\title{
Confronting AIDS
}

\author{
June E. Osborn
}

Institute of Medicine, National Academy of Sciences, Confronting AIDS: Directions for Public Health, Health Care, and Research (Washington, D.C.: National Academy Press, 1986), \$24.95

In October 1986 the Institute of Medicine and the National Academy of Sciences (IOM/NAS) jointly released a report entitled Confronting AIDS: Directions for Public Health, Health Care, and Research. The document was unique in several respects. It was the first such study co-sponsored by the two organizations; it was the first to be undertaken and completed on a "fasttrack" six-month time schedule, reflecting the urgency of the problems it addressed; and it was initiated and funded independently by IOM/NAS in order to lend weight to and ensure the credibility of its findings. The charge to the Committee assembled in March 1986 had been to develop a national strategy for coping with the AIDS epidemic and to make recommendations across the entire range of complex problems raised by the advent of the new and deadly disease.

The stark numbers that form the basis of the report speak for themselves: the United States has experienced a new epidemic viral disease for less than a decade, and yet as of October 1986 we were already confronted with more than 25,000 cases of the acquired immunodeficiency syndrome-AIDS - which is a uniformly lethal disease. In addition over roo,000 more individuals were ill with diseases not technically diagnosed as AIDS but that were the result of infection with the virus, HIV; and a conservative estimate set the number of silently infected persons at $\mathbf{r . 5}$ million or more. Furthermore, as the Committee assessed the present state of knowledge concerning epidemiology and pathogenesis, it made the strongly based prediction that ten-fold more cases of AIDS would be diagnosed within the next five years.

Those figures are startling in themselves, but their ominous significance looms still larger as the ramifications of the epidemic are measured and assessed. Because HIV is transmitted almost exclusively by sexual intercourse or by blood (including contaminated IV drug apparatus), its target population is comprised dominantly of young and middle-aged adults whose social behavior puts them in its path. The life expectancy after a diagnosis of AIDS is less than two years; so for the first time since the Great Influenza Pandemic of I91819, we must contemplate the rather sudden deletion of thousands of people in the prime of life, many of whom are trained, talented, and productive members of society. The estimated incubation period-from acquisition of viral infection to onset of the disease AIDS-is at least four to five years, which means that 80 percent of the projected 250,000 cases of AIDS that will have accrued between now and r99r will result from infections already under way. That is, those people are irretrievably infected at present and incubating their future troubles.

Estimates of the likelihood that AIDS will develop in the 1.5 million or more infected, asymptomatic individuals have been rising steadily. Even a year ago it had seemed possible that a large majority of them would remain disease-free, even though the nature of the virus infection would render them infectious for life. But the Epidemiology Working Group for the IOM/NAS study was faced with harsh new realities: as the U.S. epidemic finished its fifth year in 1986, the expected downturn in the frequency with which seropositive individuals became ill had not materialized. The incubation period estimate had been extended steadily from two to more than four years. Indeed, a few patients were illustrating a dreadful new facet of the virus' potential, for immunologic disease-for which the syndrome was named and which had been deemed the hallmark of HIV pathogenicity-was sometimes preceded and occasionally replaced by destructive, progressive disease of the central nervous system. These new insights forced a revision in thinking about prognosis, and by the time of the report 25 percent was viewed as a conservative estimate of the likelihood of disease in infected individuals; and the way was left 
open, of necessity, for further upward revision. While the report settled on an upper boundary of 50 percent for its official estimate, it was the opinion of some experienced epidemiologists in the study group that a substantial majority of infected individuals would some day suffer devastating and ultimately lethal disease resulting directly from the immunologic and/or neurologic destruction attendant on infection with HIV.

The grimness of these predictions for the individuals involved is amplified by their repercussions on the larger society. Health care costs directly resulting from AIDS are projected to escalate from a base of zero in 1977 (even in retrospect, the disease was nonexistent in the United States until that time) to $\$ 8-\$ 16$ billion annually by $199 \mathrm{I}$. This appalling figure does not include any additional cost estimates for nonAIDS illness caused by HIV, nor does it allow for innovative therapies yet to be invented. Experience to datesummarized in the report-suggests that modifications of our rigid, interventive medical care approaches and systems could improve the quality of care, as well as cutting its costs several-fold. Yet the Committee's study revealed that strategies to implement such flexible patientoriented, cost-effective care are barely on the drawing board, despite the urgency of their present need.

Nursing homes and hospices may offer far more appropriate care and solace than do intensive care units for many victims of the new virus, and yet demographic and sociological features of the epidemic result in refusal or reluctance of existing institutions to respond. Hospices are a relatively new phenomenon in health care and are still relatively scarce, while nursing homes have been designed, in large part, to meet the needs of 85 -year-old ladies of means. They are distinctly inhospitable to 35-year-old single men who have had to "spend down" to destitution during earlier stages of their illness, not to mention homeless 25-yearold addicts or prostitutes.

The medical and health care facts alone present daunting problems, but the Committee's report brought out other dimensions as well. Because the epidemic in this country has thus far focused its fury on groups such as gay men and intravenous drug abusers, who are at ready at the fringe of societal tolerance, community reactions to AIDS have sometimes posed a serious threat to the pluralism that is at the heart of our democratic system. Widespread fear has been generalized to entire groups of individuals in ways that infringe on their civil liberties. AIDS and ARC patients are at jeopardy in matters as basic as employment, insurance, housing, and educational opportunity. Terror pursues them even beyond the grave, as funeral directors balk at dealing with their remains.

These threats to the fundamental rights on which our society is based are especially worrisome because they derive largely from the unfounded fear of casual contagion, even though the reassuring facts concerning transmissibility are reinforced with each new study of the American epidemic: only sex, blood (and needles), or birth-pre- or postnatal transfer of virus to the newborn-are implicated as routes of infection. Exhaustive review of persons and situations in which other modes of spread might have occurred yield nothing: not even infection by HIV, much less AIDS, has transpired in settings as intimate as monthslong home care of loved ones dying of AIDS; sharing of razors, toothbrushes, and toilets; hospital care of yet-to-be-diagnosed infected patients; or even accidental needlepricks or lacerations with sharp instruments directly after their use in AIDS patients.

These tests of transmissibility have occurred in the natural settings of homes and hospitals, but it would have been difficult to design a more rigorous set of experiments to test the assertion that HIV is difficult to transmit. Actually, these results make good biological sense, for HIV is a lentivirus-one of a number of members of the retrovirus subgroup that are characterized by a long incubation period, a predilection for the central nervous system and for lymphoid cells, species specificity and a greatly restricted transmissibility. Therefore the data concerning the new human virus are not only convincing in their own right but also fit well with what is known about several related viruses in other species. This reassurance about poor transmissibility underscores the need to address those destructive social dynamics that are fueled by misperceptions and misplaced fear of contact contagion. The challenge to the legal system to maintain fairness in the face of widespread anxiety is a formidable one.

A special focus of the Committee's attention was the status of the blood supply, for a major, unavoidable source of risk early in the epidemic was contaminated blood and blood products. As early as March I 983 , before HIV was identified, information about risk groups led to a plea by the U.S. Public Health Service that persons at risk refrain from donation. Months later, when HIV was isolated and grown in the laboratory, rapid mobilization of technology yielded a reliable, sensitive screening system for evidence of viral infection in blood and plasma. Since 1985 that system has been in place at blood centers throughout the country.

As a result of this two-pronged approach, the risk of blood transmission of HIV has been dramatically reduced, although not to zero. The Committee assessed the residual chance of acquiring HIV from a unit of blood in the U.S. as $\mathrm{I}: 100,000$, far below the risks posed by most clinical conditions in which transfusion is indicated. The direct benefits 
of knowing the blood supply is safe are obvious, and there are indirect benefits as well. It is now possible to focus public health efforts on education to facilitate prevention of further virus spread by individual decision-making about avoidance of risky behavior.

In that regard we are fortunate, for in other parts of the world the strategy of avoidance is not so easy to adopt. In parts of Africa, the scale of the epidemic is approaching truly disruptive levels as substantial fractions of adult populations are involved. There is real disaster in the offing for efforts at medical care of AIDS patients in such countries, where the annual per capita investment in health care is often less than the cost of a single AIDS-antibody screening test. That something must be done quickly is underscored by the increasingly ominous reports of infection in 10 percent or more of adult populations in parts of Central and East Africa, but even fundamental issues of transmissibility are obscured in those areas where "injectionism" and heterosexual promiscuity complicate efforts at prevention of further spread. What is clear is that the blood supply is far from secure in such areas, and both donation and usage patterns underscore the benefits of having achieved quick control of that facet of HIV's spread.

While African countries and the United States have experienced in creasing epidemic involvement since the early I980s, some parts of the world, especially Asia, are still relatively unaffected. Most countries in Europe and in the Western hemisphere have begun to experience the rapidly expanding onslaught of AIDS, with epidemic patterns more reminiscent of the U.S. than of Africa. The prominent role of intravenous drug abuse as a risk behavio in several countries, such as Italy and Sweden, underscores the potential danger posed in the U.S. by the estimated 750,000 IV heroin addicts plus at least an equal number of persons who experiment with IV drugs on an intermittent basis. The fact that 60 percent or more of New York City addicts are already infected, for example, lends urgency to the need to expand our overtaxed treatment capacity to deal with addiction. The fact that other large metropolitan areas, such as Los Angeles, have yet to see much dissemination of HIV among addicts suggests that timely intervention could reap large rewards in terms of prevention.

The IV drug abuse issue is especially worrisome in that many health care innovations to date have been predominantly designed by and for gay males. Efforts to deal with addicts in the same terms have resulted in much frustration. Furthermore, the heterosexual partners of IV drug abusers have illustrated, from the beginning of the epidemic, that homosexual practices are not the only route of sexual spread of the virus. The study group predicted that 90 percent of pediatric AIDS will occur in infants born into drug-afflicted families and infected in utero or in the perinatal period. Thus the special horrors of AIDS as it occurs clinically will be compounded by parental drug abuse and/or orphan status as more and more perinatally infected infants survive and undergo progressive virus-mediated damage to their nervous and immune systems.

The fundamental charge to the IOM/NAS Committee, as noted earlier, was to delineate a national strategy for AIDS. While the rapid evolution of the problems established some natural limits to the precision of planning, serious effort was made in the report to point out the pathways that are open for promising progress and those that are especially forbidding.

First and foremost, the Committee urged continuation of the intense and fruitful endeavors in biomedical research. The clarity with which many of the above assessments and prognostications were made derived from the rapid exploitation of a rich pre-existing base of fundamental knowledge in immunology, virology, and molecular biology. Just as important, the insights needed to further define and pursue leads that will ultimately facilitate effective vaccine and treatment strategies will require further advances. It is likely that many of these will derive from broad progress on the basic science front rather than from sharply targeted assaults on specific problems. The Committee noted the contributions of epidemiology to our understanding of the new virus and its pathogenic behavior, and underscored the need for continuing epidemiologic investigations, both to address questions still outstanding and to remain alert for new insights or change.

Despite the past triumphs and ongoing need for biomedical research, however, the Committee concluded that neither vaccine nor therapeutic cure is likely to emerge as a major useful strategy within the next five years. This appraisal was arrived at after much careful discussion and assessment of possible avenues of approach. It is worth noting that the Committee included several of the scientists whose research had led to the current advanced state of knowledge concerning the human retrovirus. Their opinion should not be undervalued as pessimism, nor should it be received with nihilistic resignation. Rather, it was a sober judgment arrived at after review of the extraordinary difficulties attendant on achieving either a vaccine or a therapeutic strategy for dealing with HIV.

In the realm of immunization, notwithstanding the impressive feats of recent decades that have banished such scourges as polio and measles, there are no examples yet developed of useful vaccines for chronic viral 
infections in which antibodies do not play a dominant defensive role in host response. The new retrovirus that causes AIDS establishes a lifelong infection in its host, and there is a strong suggestion from developing data that antibodies do not correlate with protection from illness. Furthermore, confirmatory warnings of difficulty come from the study of other retrovirus infections in species of veterinary interest, where vaccine efforts to date have met with marginal success at best.

Some of the predicted problems arise from the fact that the rapid mutability of HIV's genetic message results in what is termed antigenic variation. This genetic plasticity might render a vaccine strain of virus obsolete in the face of new variants. The paucity of animal models presents another hurdle in the way of both vaccine and treatment development. While several primate species have been found to have their own AIDS-like retroviral infections, only chimpanzees, among potential host species, can be infected with HIV. But the chimpanzee is an endangered species, and the absolute number of animals available for study is extraordinarily limited and must be rationed with great deliberation and care. Finally, even if an ideal vaccine candidate were at hand, time constraints intrinsic to the design and evaluation of vaccine trials for a disease with a four- to five-year incubation period would mean that a mass strategy for prevention by immunization would be years off.

Efforts at treatment with antiviral drugs are inherently complicated because of the intimate nature of HIV infection; once established, the viral genetic message is durably entwined with the genes of host cells in such a way that lifelong therapy would almost surely be necessary to maintain suppression of the virus. The pace of progress in biomedical spheres is so rapid that the new drug AZT emerged as a promising agent after most of the text of the report had been written. In the blush of promise that prevailed at the time the study results were announced, the final draft was modified somewhat to hedge the gloomy predictions that development of effective therapeutic agents might be more than five years off. Yet the Committee's initial attitude of caution appears to have been well founded. As further experience develops, the prolongation of life in AZT-treated patients-which led federal officials to discontinue placebo-controlled experiments on ethical groundsturns out to be achieved at the cost of an extraordinarily high rate of transfusion-dependency resulting from hematotoxicity.

Thus the conclusion that biomedical miracles should not be expected to provide a quick solution to this problem is, if anything, reinforced by the AZT experience. It is no accident that antiviral therapy has been so long in coming, in contrast to antibacterial drugs. Viral infections are so parasitic in their commandeering of host cell functions to reproduce themselves that any chemical defense must attack processes that may be integral to the host's good health. That truism is even more pertinent in the context of retroviruses, where the initial event of infection usually splices viral genetic information right into the host's own DNA, and subsequent viral production is intimately linked to host-cell control mechanisms. In such circumstances treatment must almost surely be sustained over months or years, perhaps for the lifetime of the host. Thus, drugs that could serve the million or more infected individuals would need to be exceptionally non-toxic, not to mention inexpensive, in order to serve a useful public health purpose.

The long road to biomedical "breakthrough" must be traveled with a sense of urgency. By the same token, however, the Commit- tee noted a great need for parallel research efforts in social and behavioral sciences to facilitate community and national efforts to interrupt the spread of the epidemic. Indeed, it was the unpromising prospect of cure or vaccine development, coupled with the poor transmissibility of the virus, that led the Committee to its most striking conclusion: that education was likely to be the cornerstone of our response to the AIDS threat for years to come. They noted that practical means of prevention-avoidance of unsafe sex practices, use of barrier devices such as condoms, and avoidance of intravenous drugs and/or contaminated drug paraphernalia - were ready at hand and should be mobilized as rapidly as possible. To this end it was recommended that for every dollar spent on biomedical research (and a research funding level of $\$ \mathrm{I}$ billion was called for by 1990), an equal sum should be invested in preventive education and public health endeavors to communicate what we already know and to minimize the further spread of HIV. This call for an intensive educational campaign advocated the frank and detailed explanation of the sexual modes of spread and proposed utilization of a wide variety of media in order to reach the heterogeneous social and economic groups at risk.

It is not possible in a brief commentary to convey the breadth of issues tackled in the IOM/NAS study. For instance, the Committee report included a detailed discussion of the ethical issues raised by public health concerns. The group took a firm stance against mandatory screening programs and urged that serological testing for HIV antibodies be available on a strictly confidential basis in the context of pre- and post-test counseling. The report suggested that the issues of insurance and of health care financing were so complex that special task forces should be established quickly to address 
them. It also advocated substantial involvement in international health problems posed by the epidemic, with particular emphasis on cooperation with international agencies such as the World Health Organization.

Finally, the Committee concluded that the potential breadth of impact of the epidemic warranted creation of an ongoing commission with either presidential or presidentialcongressional mandate to help coordinate the efforts of the varied sectors of society that would be pressed to respond to the disease. Since government, industry, and academia all must play distinctive roles in order to maximize the effective re- sponses required in the predicted crisis, the proposed commission was felt to be an important mechanism whereby these components could be orchestrated and stimulated to interact successfully.

The impact of the 1OM/NAS report remains to be seen. It is encouraging to note that in the days immediately preceding its release, the surgeon general of the United States took a strong stance of advocacy for early sex education of children in the schools-a muchneeded component of the educational program envisioned by the Committee. It is perhaps too soon to bemoan the fact that the called-for studies of insurance and health care financing, and the establishment of a Commission have not yet evoked a response; but it is also difficult to be patient. The "duty to warn" seems increasingly imperative as the fearful finality of AIDS becomes ever clearer. The estimated size of the infected population is so large already that one or two more doublings could take the social scope of the problem beyond our ability to cope humanely with its consequences. The sense of urgency conveyed so clearly by the IOM/NAS Committee must be maintained. The price of bureaucratic inertia in this unique period of human history would be intolerably high. 